\title{
Metabolic Profiling of Saliva in Patients with Primary Sjögren's syndrome
}

Jopi JW Mikkonen', Maria Herrala², Pasi Soininen ${ }^{3}$, Reijo Lappalainen ${ }^{1}$, Leo Tjäderhane ${ }^{4,5}$, Hubertus Seitsalo ${ }^{6}$, Raija Niemelä7, Tuula Salo ${ }^{2,5,8}$, Arja M Kullaa ${ }^{2,9}$ and Sami Myllymaa ${ }^{1}$

${ }^{1}$ SIB Labs and Department of Applied Physics, Faculty of Science and Forestry, University of Eastern Finland, Kuopio, Finland

${ }^{2}$ Department of Diagnostics and Oral Medicine, Institute of Dentistry, University of Oulu, Finland

${ }^{3}$ School of Pharmacy, Faculty of Health Sciences, University of Eastern Finland, Kuopio, Finland

${ }^{4}$ Department of Pedodontics, Cariology and Endodontolgy, Institute of Dentistry, Universities of Oulu and Turku, Finland

${ }^{5}$ Department of Oral Diseases, Oulu University Hospital, Oulu, Finland

${ }^{6}$ Dental Clinic Medone, Helsinki, Finland

${ }^{7}$ Department of Rheumatology, Oulu University Hospital, Oulu, Finland

${ }^{8}$ Institute of Dentistry, University of Helsinki, Helsinki, Finland

${ }^{9}$ Educational Dental Clinic, Health Centre of Oulu, Oulu, Finland

\section{Abstract}

Objective: To investigate the feasibility of ${ }^{1} \mathrm{H}-\mathrm{NMR}$ spectroscopy for metabolic profiling of human saliva samples and to determine whether the concentration of certain salivary metabolites, mainly representing small organic acids and amino acids, differ between patients with primary Sjögren's syndrome (pSS) and healthy controls.

Methods: Stimulated whole-mouth saliva (SWMS) was collected from female pSS patients $(n=15$, all fulfilling the revised European Community proposed criteria). Salivary flow rate was immediately determined, samples were then frozen and subsequently analyzed by ${ }^{1} \mathrm{H}-\mathrm{NMR}$ spectroscopy in comparison with samples collected from healthy individuals $(n=15)$

Results: From each sample, up to 24 metabolites could be identified and quantified. Choline and taurine concentrations were very significantly higher in the pSS patients compared to healthy controls $(p<0.001)$, but their concentrations correlated negatively with salivary flow rate. Alanine and glycine concentrations were significantly higher $(p=0.004, p=0.007$, respectively), whereas butyrate $(p=0.034)$, phenylalanine $(p=0.026)$ and proline $(p=0.032)$ were only slightly higher in pSS saliva samples than in controls.

Conclusions: NMR spectroscopy has a potential for quantitative metabolic profiling of saliva samples. NMR spectroscopy is suitable for the analysis of NAAs in saliva and it can bypass the other methods, which are normally suitable for analysis of just one metabolite.

Keywords: Saliva; Metabolomics; NMR spectroscopy; Amino acid neurotransmitters; Biomarkers; Sjögren's syndrome

\section{Introduction}

Sjögren's syndrome(SS) is an autoimmune rheumatic disease which causes chronic inflammation in the exocrine glands. Salivary glands' hypofunction is a consequence of ductal and acinar cell destruction and causes lower salivary secretion [1]. Sjogren's syndrome manifests in patients in two different forms: primary (pSS) and secondary (sSS). sSS usually is a consequence of some other rheumatic disease, for example systemic lupus erythematosus (SLE) or rheumatoid arthritis (RA). pSS is otherwise already a disease by itself [2]. SS occurs worldwide with similar prevalence $(0.5-1.5 \%)$ and around $90 \%$ of the patients are female. Typical age of the patients is between 40 and 60 years, but the disease also exists in all age groups [1]. Patients who suffer from SS have often a serious malfunction of exocrine glands, sometimes it is called the 'sicca syndrome'. The main clinical features are xerostomia, 'dry mouth 'and xerophthalmia, 'dry eyes' [3]. SS can affect also exocrine glands in esophagus, stomach, bowel, pancreas and bladder [4].

The pathogenesis of SS is complex and still partially unknown. Many factors, as genetic, hormonal, environmental, innate and adaptive immunity and the autonomic nervous system, have been thought to be involved in the pathogenesis of SS. The diagnostic criteria for SS has been presented by the American-European Consensus Group where the classification criteria includes the six main clinical findings; defining also separate diagnostic standards for pSS and sSS [5].

Recent advances in metabolic profiling techniques offer a powerful and promising approach to identify biomarkers associated with several disorders such as celiac disease [6,7], leukemia [8], breast cancer $[9,10]$ and oral carcinomas [11]. A variety of different analytical techniques have been used in the metabolic profiling studies: currently the mass spectrometry (MS) has been utilized most frequently. Among MS techniques, especially two-dimensional gel electrophoresis (2D-GE) and matrix-assisted laser desorption ionization time-of-flight (MALDITOF) have mostly been applied to analyze SS saliva samples [12-16].

Instead, another powerful metabolic profiling technique, namely nuclear magnetic resonance (NMR) spectroscopy, has remained largely underexplored in saliva analysis. Although some salivary metabolites have been successfully identified and inter- and intrasubject variability has been investigated by using ${ }^{1} \mathrm{H}$ NMR [17-21] or ${ }^{13} \mathrm{C}$ NMR spectroscopy [22], well-designed studies aiming to biomarker identification associated to certain health disorder are very rare [23].

*Corresponding author: Arja M Kullaa, Department of Diagnostics and Ora Medicine, Institute of Dentistry, Faculty of Medicine, University of Oulu, Aapistie 3, SF-90220 Oulu, Finland; Tel: +358-40-1426319; E-mail: arja.kullaa@oulu.fi

Received October 23, 2013; Accepted November 26, 2013; Published November 29, 2013

Citation: Mikkonen JJW, Herrala M, Soininen P, Lappalainen R, et al. (2013) Metabolic Profiling of Saliva in Patients with Primary Sjögren's syndrome. Metabolomics 3: 128. doi:10.4172/2153-0769.1000128

Copyright: @ 2013 Mikkonen JJW, et al. This is an open-access article distributed under the terms of the Creative Commons Attribution License, which permits unrestricted use, distribution, and reproduction in any medium, provided the original author and source are credited. 
The purpose of this study was to investigate the feasibility of ${ }^{1} \mathrm{H}-\mathrm{NMR}$ spectroscopy for metabolic profiling of human saliva samples and to clarify the potential of NMR spectroscopy for detecting the salivary metabolites associated with pSS. To the best of our knowledge, this study, for the first time, establishes the composition and concentration of metabolites in saliva samples collected from welldefined pSS patients and healthy control individuals.

\section{Material and Methods}

The pSS patients were around the Oulu area and had been diagnosed at the Department of Rheumatology, Oulu University Hospital, Oulu, Finland by RN. Detailed description of the patient inclusion and exclusion criteria has been presented previously by Niemelä et al. [24]. Briefly, all patients were required to fulfill the revised criteria of SS made by European Community [5]. Pregnant patients and patients with anticoagulant medication were left out. A total of 44 patients were screened, of which 22 participated in a study where saliva samples were collected [25]. The patients underwent an oral and dental examination. Exclusion criteria included smoking habits and oral or systemic diseases except pSS. Thus saliva samples of 15 female patients were available for the NMR analysis. Patients' ages varied between 28 and 68 years (mean age 48.6 years), and the time between the diagnosis of SS and the beginning of the study varied between 4 and 23 years. The control group contains 15 healthy, non-smoking female persons, age range $28-68$ years (mean age 49.8 years). None of these subjects had any chronic disease or received any treatment regularly that could alter the salivary test results. The Oulu University Hospital Ethical Committee gave their approval for this study. All the patients gave their written consent according to the Declaration of Helsinki.

\section{Collection of saliva samples}

Saliva samples were collected using standard techniques according to Navazesh [26]. Briefly, stimulated whole-mouth saliva (SWMS) was collected in the morning (between 10 and $12 \mathrm{am}$ ) in order to assess any circadian effect. All subjects were asked to abstain from eating and drinking for at least $1 \mathrm{~h}$ before sample collection. Collection of stimulated saliva started with a stimulation time of 30 seconds by chewing paraffin wax (1 g; Orion Diagnostica, Espoo, Finland). Each subject was instructed to tilt their head forward, allow saliva to collect in the mouth, and let the saliva drain into a sterile $20 \mathrm{ml}$ weighed polypropylene tube for $5 \mathrm{~min}$. The saliva flow rate $(\mathrm{ml} / \mathrm{min})$ was measured immediately after saliva collection. Saliva samples were transported to the laboratory on ice and centrifuged at 3,000xg for 20 $\min$ at $4^{\circ} \mathrm{C}$. The supernatants were stored at $-20^{\circ} \mathrm{C}$ for later use.

\section{Sample preparation}

$450 \mu$ l of each saliva sample was thoroughly mixed with $50 \mu$ lof NMRbuffer (1.5 $\mathrm{M} \mathrm{KH}_{2} \mathrm{PO}_{4}, 2 \mathrm{mM} \mathrm{NaN}_{3}, 5.8 \mathrm{mM}$ sodium 3-(trimethylsilyl) propionate-2,2,3,3- $\left.\mathrm{d}_{4}, \mathrm{D}_{2} \mathrm{O}, \mathrm{pH} 7.4\right)$ and subsequently centrifuged at $10,000 \times \mathrm{g}$ for $5 \mathrm{~min}$ at $+4^{\circ} \mathrm{C}$ to remove any solid debris. The resulting supernatant was transferred to $5 \mathrm{~mm}$ NMR tubes.

\section{Data acquisition}

The high-resolution 1D ${ }^{1}$ Hwere acquired using a Bruker AVANCE III $\mathrm{HD}$ spectrometer operating at $600.20 \mathrm{MHz}\left({ }^{1} \mathrm{H}\right.$ observation frequency) and equipped with an inverse triple resonance Bruker CryoProbe Prodigy $5 \mathrm{~mm}$ probe head including ATM and Z-gradient coil. The spectrometer was controlled via TopSpin 3.2 (Bruker BioSpin $\mathrm{GmbH}$ ) software. The samples were stored at $+6^{\circ} \mathrm{C}$ in the sample changer until the measurement and were preheated to $+25^{\circ} \mathrm{C}$ ca. $30 \mathrm{~min}$ prior to the measurement. Each sample was shimmed automatically using topshim routine. The $1 \mathrm{D}^{1} \mathrm{H}$ data (64k data points) were recorded using a $\mathrm{T}_{2}$-relaxation-filtered pulse sequence) that suppress most of the broad macromolecule signals at $+25^{\circ} \mathrm{C}$ with a $6.8 \mathrm{~s}$ repetition time (relaxation time $4.0 \mathrm{~s}$ and acquisition time $2.8 \mathrm{~s}$ ) after 8 dummy scans using 96 transients with an automatically calibrated $90^{\circ}$ pulse to achieve the required signal-to-noise level. A Bruker cpmgld pulse sequence with $\mathrm{T}_{2}$-filter time of $80 \mathrm{~ms}$ and irradiation field of $50 \mathrm{~Hz}$ to suppress the water peak was used. The $90^{\circ}$ pulse was calibrated automatically for each sample. A constant receiver gain setting was used for all the samples.

\section{Data processing}

The acquired spectra were processed and phase corrected manually (TopSpin 3.0, Bruker BioSpin GmbH). Prior to the Fourier transformations to spectra, the measured free induction decays were multiplied with an exponential window function with a $1.0 \mathrm{~Hz}$ line broadening. The metabolites were identified by referring to the published data [22]. In total, 24 metabolites could be identified for quantification. Quantification of the metabolites was done using the constrained total-line-shape fitting type approach of the PERCH NMR software (PERCH Solutions Ltd, Kuopio, Finland), which allows an accurate quantification of identified metabolites even if the signals are overlapping or the baseline is not linear due to heavy protein background envelope [27]. The signal areas were referenced to an internal reference compound (TSP), which had a known concentration. The final concentrations are reported as $\mu \mathrm{mol} / \mathrm{l}$ in saliva.

\section{Statistical analysis}

Metabolite levels are expressed as mean \pm SD in tables and as box plots in figures.

The Shapiro-Wilk test and the values of kurtosis and skewness were used to analyze the data for normality distribution. Student's t-test was used to compare stimulated salivary metabolite concentrations between pSS patient and healthy control groups.

Correlation were analyzed using Spearman's test. SPSS software, version 19.0 (SPSS Inc., Chicago, IL, USA) was used in all analyses. Significance was set at $\mathrm{P}<0.05$.

\section{Results}

Representative ${ }^{1} \mathrm{H}$-NMR spectra of saliva samples collected from a healthy participant and a pSS patient are shown in Figure 1. Up to 24 metabolites were assigned in each sample including organic acids (acetate, butyrate, citrate, formate, lactate, propionate, succinate), carbohydrates (butanol, ethanol, fucose, isopropanol, galactose, glucose, methanol), amino acids (alanine, glycine, histidine, phenylalanine, taurine, tyrosine) as well as amines (choline, methylamine, proline, trimethylamine). Some metabolites, e.g. leucine, valine and isoleucine, were observed in only a few samples, and thus excluded from data analysis. The metabolite concentrations are presented in Table 1 . The concentration of choline and taurine was significantly higher $(\mathrm{p}<0.001)$ in the pSS patients ( $\mathrm{n}=15$, all females, aged 28 to 68 years) compared to the healthy controls ( $\mathrm{n}=15$, all females, aged 28 to 68 years) (Figure 2 ). Moreover, alanine and glycine were significantly higher $(\mathrm{p}=0.004$, $\mathrm{p}=0.007$, respectively) in concentration in the pSS group. Butyrate $(\mathrm{p}=0.034)$, phenylalanine $(\mathrm{p}=0.026)$ and proline $(\mathrm{p}=0.032)$ were only slightly higher in SS saliva samples than in controls. Regarding other 
metabolite concentrations, no statistically significant differences were found.

Finally we studied the associations between salivary flow rate and the concentration of metabolites. Salivary flow rate was significantly lower in the pSS group $(0.82 \pm 0.29 \mathrm{ml} / \mathrm{min})$ than in the controls $(1.99$ $\pm 0.51 \mathrm{ml} / \mathrm{min}, \mathrm{p}<0.001)$. The concentration of choline $(\mathrm{r}=-0.57$, $\mathrm{p}=0.001)$ and taurine $(\mathrm{r}=-0.50, \mathrm{p}=0.006)$ increases when the salivary flow rate decreases (Figure 3 ). Other metabolites did not significantly correlate with salivary flow rate.

\section{Discussion}

Saliva provides a unique window into the biological processes and functioning of the human body. It has been shown that saliva reflects the spectrum of health and disease states [28] and has therefore been labeled as "a mirror of the body" [29]. Most compounds found in blood are also present in saliva, but usually in lower concentrations [30]. Therefore, there have been concerns that the low concentrations of analytes in saliva may prevent salivary diagnostics from being clinically accurate enough. However, with the recent development of saliva collection methods, storage of samples and particularly new highly sensitive analysis techniques, low compound concentrations are no longer a limitation. NMR spectroscopy poses several advantages over classical biochemical assays. It represents a reproducible technique for investigating the salivary metabolities without extensive sample preparation, and the measurements are detectable in as little as 450 $\mu \mathrm{l}$ of saliva. Furthermore, NMR spectra provide information about physicochemical status of certain salivary metabolites.

Since the pSS is usually diagnosed only after advanced gland tissue

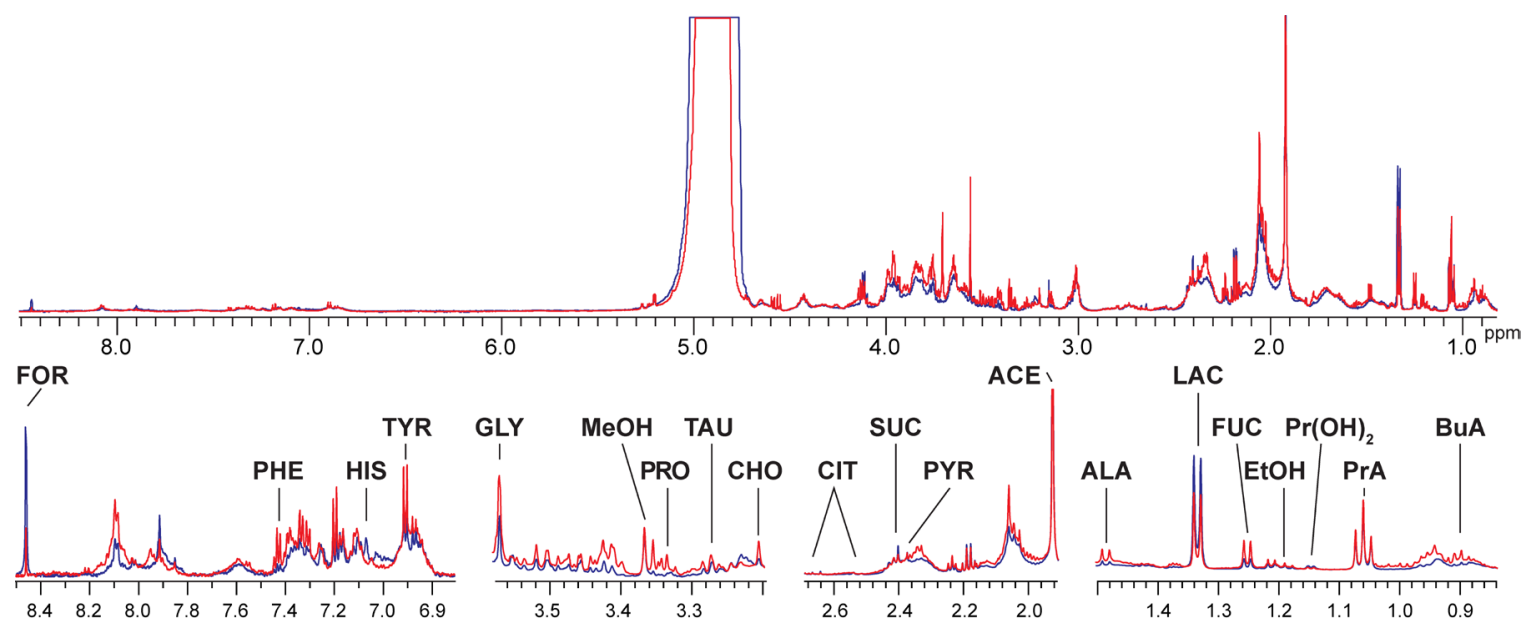

Figure 1: Spectral characteristics and metabolic content of a typical saliva $600 \mathrm{MHz} 1 \mathrm{H}$ NMR spectrum of a patient with primary Sjögren's syndrome (red) and a healthy control (blue). FOR, formate; PHE, phenylalanine; HIS, histidine; TYR, tyrosine; GLY, glysine; MeOH, methanol; PRO proline; TAU, taurine; CHO, choline; CIT, citrate; SUC, succinate; PYR, pyruvic acid; ACE, acetate; ALA, alanine; LAC, lactate; EtOH, ethanol; $\operatorname{Pr}(\mathrm{OH}) 2$, isopropanol; PrA, propanoic acid; BuA, butyric acid.
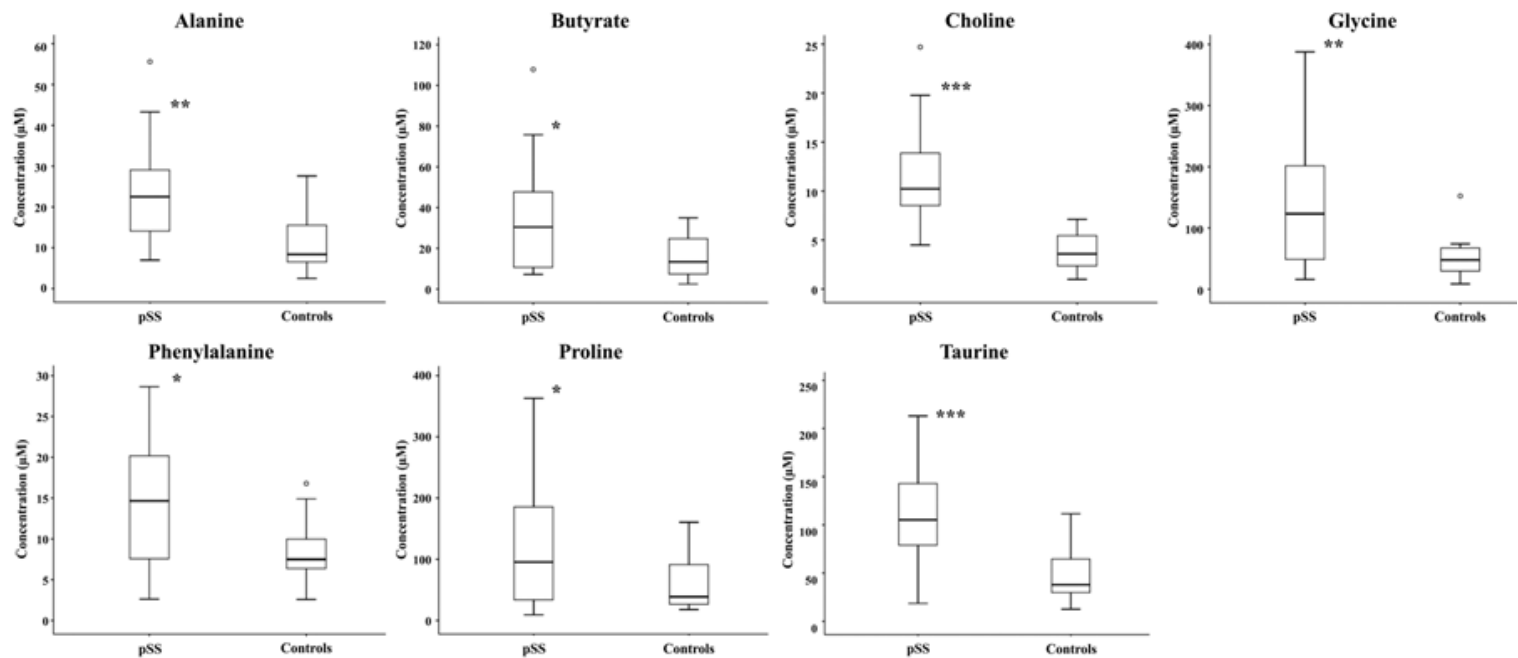

Figure 2: Box- and whisker plots illustrating concentrations of some of the measured salivary metabolites in primary Sjogren's syndrome $(p S S)$ patients $(n=15)$ relative to healthy controls $(n=15)$. The median is depicted with a horizontal line in the middle portion of the box. The bottom and top boundaries of the boxes represent the first and third quartiles, respectively, and whiskers represent 95th and 5th percentiles. Significant differences were calculated using Mann-Whitney U-test $\left({ }^{*} \mathrm{P}<0.05 ;{ }^{* *} \mathrm{P}<0.01 ;{ }^{* *} \mathrm{P}<0.001\right)$ 
Citation: Mikkonen JJW, Herrala M, Soininen P, Lappalainen R, et al. (2013) Metabolic Profiling of Saliva in Patients with Primary Sjögren's syndrome. Metabolomics 3: 128. doi:10.4172/2153-0769.100012

Page 4 of 6

\begin{tabular}{|c|c|c|c|c|c|}
\hline \multicolumn{3}{|c|}{ pSS patients $(n=15)$} & \multicolumn{3}{|c|}{ Controls $(n=15)$} \\
\hline \multirow{2}{*}{ Metabolite } & \multicolumn{2}{|c|}{ Concentration $(\mu \mathrm{M})$} & \multicolumn{2}{|c|}{ Concentration $(\mu \mathrm{M})$} & \multirow[b]{2}{*}{ P value ${ }^{(a)}$} \\
\hline & Mean \pm S.D & Range & $P$ value ${ }^{(a)}$ & Range & \\
\hline Acetate & $1482.7 \pm 952.6$ & $202.7-3676.6$ & $1503.0 \pm 715.2$ & $670.6-3035.2$ & 0.948 \\
\hline Alanine & $23.7 \pm 13.2$ & $7.0-55.6$ & $11.4 \pm 7.3$ & $2.5-27.6$ & $0.004^{* *}$ \\
\hline Butanol & $14.1 \pm 12.1$ & $1.5-45.1$ & $8.6 \pm 5.8$ & $0.8-20.7$ & 0.126 \\
\hline Butyrate & $34.7 \pm 29.0$ & $7.2-107.8$ & $16.4 \pm 10.9$ & $2.5-34.9$ & $0.034^{*}$ \\
\hline Choline & $11.4 \pm 5.3$ & $4.5-24.7$ & $4.1 \pm 2.0$ & $1.0-7.1$ & $<0.001^{* * *}$ \\
\hline Citrate & $18.3 \pm 7.9$ & $7.2-34.4$ & $18.3 \pm 13.8$ & $3.4-46.5$ & 0.996 \\
\hline Ethanol & $25.4 \pm 12.8$ & $7.2-57.7$ & $26.3 \pm 59.6$ & $7.9-241.3$ & 0.956 \\
\hline Formate & $152.0 \pm 195$ & $6.3-660.8$ & $80.1 \pm 63.3$ & $3.3-220.5$ & 0.192 \\
\hline Fucose & $46.5 \pm 35.9$ & $3.7-113.6$ & $66.3 \pm 40.3$ & $20.2-150.5$ & 0.167 \\
\hline Glycine & $150.9 \pm 120.5$ & $16.0-387.7$ & $51.5 \pm 35.4$ & $8.7-152.3$ & $0.007^{\star *}$ \\
\hline Histidine & $30.3 \pm 13.9$ & $8.7-53.8$ & - & - & - \\
\hline Isopropanol & $1.84 \pm 0.8$ & $0.2-3.7$ & - & - & - \\
\hline Lactate & $265.6 \pm 140.4$ & $25.4-509.7$ & $198.3 \pm 174.5$ & $14.1-649.0$ & 0.255 \\
\hline Methanol & $28.5 \pm 17.5$ & $9.7-65.6$ & $27.7 \pm 9.2$ & $8.3-43.9$ & 0.878 \\
\hline Methylamine & $2.1 \pm 1.7$ & $0.5-5.7$ & $2.0 \pm 0.9$ & $1.1-3.8$ & 0.818 \\
\hline Phenylalanine & $14.3 \pm 8.4$ & $2.6-28.6$ & $8.5 \pm 3.6$ & $2.6-16.8$ & $0.026^{*}$ \\
\hline Proline & $133.5 \pm 114.5$ & $9.1-362.9$ & $59.1 \pm 44.1$ & $17.8-160.5$ & $0.032^{*}$ \\
\hline Propionate & $284.3 \pm 205.7$ & $49.8-722.1$ & $235.7 \pm 156.2$ & $69.0-621.9$ & 0.472 \\
\hline Pyruvic acid & $17.6 \pm 11.2$ & $0.9-37.5$ & $10.4 \pm 7.8$ & $0.0-24.3$ & 0.050 \\
\hline Succinate & $33.8 \pm 32.1$ & $6.7-131.8$ & $28.2 \pm 15.7$ & $5.3-49.5$ & 0.549 \\
\hline Taurine & $110.5 \pm 49.6$ & $18.6-213.0$ & $48.3 \pm 29.0$ & $12.5-111.5$ & $<0.001^{* * *}$ \\
\hline Trimethylamine & $1.4 \pm 1.4$ & $0.1-4.9$ & - & - & - \\
\hline Tyrosine & $26.7 \pm 18.5$ & $5.1-75.4$ & $19.6 \pm 11.4$ & $6.4-50.5$ & 0.215 \\
\hline 1,2-propanediol & $40.1 \pm 71.4$ & $9.2-290.0$ & $27.3 \pm 14.2$ & $9.8-59.0$ & 0.500 \\
\hline
\end{tabular}

(a)Student's t-test; $p<0.001^{* * *} ; p<0.01^{* *} ; p<0.05^{*}$

Table 1: Comparison of salivary metabolite concentrations between primary Sjögren's syndrome (pSS) patients and healthy controls.
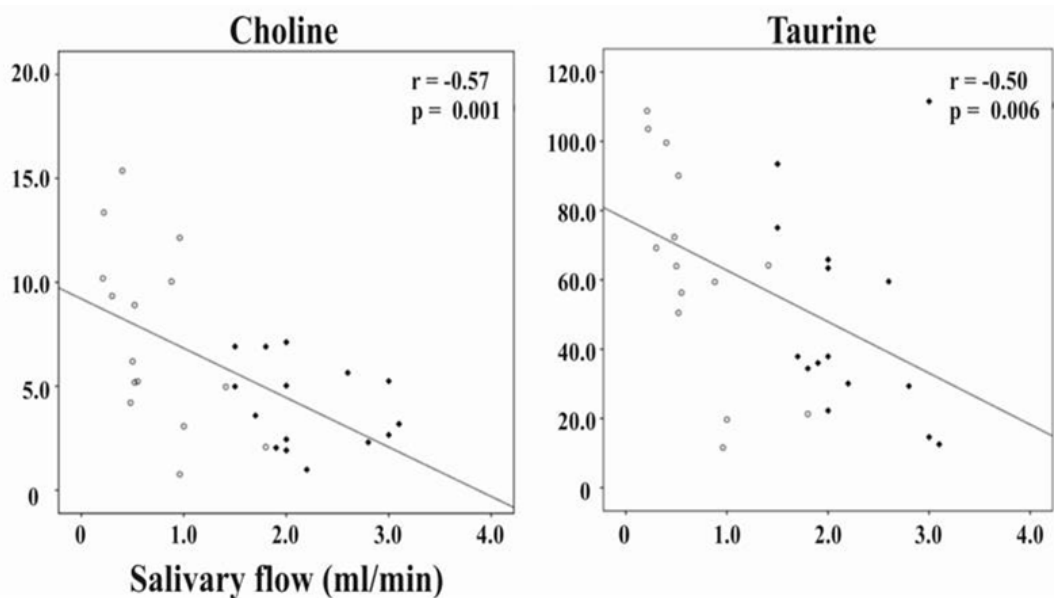

Figure 3: The correlations between the salivary flow rate and concentration of choline and taurine. pSS patients $(n=15)$ are marked by open circles and healthy controls $(n=15)$ by black squares. The solid line represents the linear regression curve of the best fit. $r=$ Spearman's correlation coefficient.

destruction has already occurred, sensitive and specific early diagnostic methods to screen the pSS patients before the development of subjective symptoms are needed [31]. Salivary metabolic provides meaningful information about pSS. This study demonstrates the quantitative analysis of salivary metabolites in stimulated saliva of pSS patients and healthy subjects, using ${ }^{1} \mathrm{H}$ NMR spectroscopy. The significant differences in several metabolite concentrations between the patients with pSS and controls could be observed. However, since the range of measured metabolite concentrations considerably overlap between the pSS patients versus healthy controls, it feels impossible to discriminate patients from controls. Therefore, it seems that the discriminate power needs to be enhanced by incorporating a combination of different metabolic biomarkers rather than a single marker.

Stimulated saliva is produced primarily by the major salivary glands and the metabolites of stimulated saliva may describe the impaired secretion process of the salivary glands [32]. We managed to identify and quantify over 24 metabolites from stimulated saliva samples. Some of these metabolites, i.e. choline, taurine, alanine, phenylalanine and glycine exhibited significantly higher concentration in the pSS patients' saliva than in the control group. Interestingly, all 
these metabolites are among the amino acids with important roles as neurotransmitters, which have been the focus of increasingly intense in biomedical research in recent years. Furthermore, these metabolites are associated to muscarinic-M3 receptors which play a key role in regulating salivary flow [33]. Previously, it has been suggested that deficient neural regulation of the secretory process might exist [34] This study demonstrated that concentrations of certain amino acids, i.e. choline and taurine in saliva are strongly associated with changes in salivary flow.

Many methods have been developed for the detection of amino acids [35-37], but in this study we showed that the neurotransmitter amino acids (NAAs) can be identified in saliva with NMR method. However the role of NAAs in saliva is still unknown, although changes in NAA levels in other biological fluids may correlate with a number of neurological diseases such as Alzheimer's [38] and Parkinson's diseases [39]. The NMR metabolomics can contribute to better understanding of the molecular mechanism of Sjögren's syndrome. Detection of neurotransmitters and other metabolites in saliva may add information on the pathogenesis of pSS and neural destruction of salivary glands.

This study suggests that it is possible to measure the levels of diseasespecific metabolic components of saliva using ${ }^{1} \mathrm{H}-\mathrm{NMR}$ spectroscopy. In the diagnostics based on salivary metabolites, it is clear that a combination pattern of several biomarkers but not a single one may define a specific disease [29]. Specific biomarkers for SS could possibly provide preindication of the syndrome, and be used to monitor the severity of the disease. This promising potential of analyzing the level of specific salivary metabolites has given us a clearer idea to study the role of salivary NAAs in the oral defense mechanism. However, further comparisons should be made between SS and other (autoimmune) disorders to reveal and refine the validity of these metabolic biomarkers for the diagnostics of SS.

Despite significant advantages, there are also some limitations in use of NMR spectroscopy as a screening tool. A modern NMR spectrometer uses superconducting magnets that must be cooled with liquid helium making them very complex and expensive machines to purchase and operate. In addition, performing measurements and interpretation of acquired spectra are somewhat challenging and timeconsuming. Also relatively large saliva sample volume is typically needed to measurement that may be challenging in case of dry mouth patients. However, future technology development (e.g. picoSpin ${ }^{\mathrm{Tm}}$ miniature NMR spectrosmeter, Thermo Scientific) combined with more robust data analysis tools will help to overcome present limitations.

In conclusion, our data suggest that changed concentration of certain metabolites detected in SS saliva reflects the functional impairment of the secretory processes observed in this syndrome. The results presented here should be tested in larger patient cohorts in order to reveal if the NMR spectroscopy technique suits for noninvasive screening and monitoring of the severity of SS. Due to the findings of this study, NMR spectroscopy is suitable for the analysis of NAAs in saliva and it can bypass the other methods, which are normally suitable for analysis of just one metabolite.

\section{Acknowledgements}

The authors would like to thank MSc Paula Pesonen for consultation of statistical analysis. This work was supported in part by the Finnish Funding Agency for Technology and Innovation (Tekes) project Oraldent 2186/31/2010.

\section{References}

1. Jonsson R, Vogelsang $P$, Volchenkov R, et al. (2011)The complexity of Sjögren's syndrome: novel aspects pathogenesis. ImmunolLett 141: 1-9.
2. Amarasen R, Bowman S (2007) Sjögren's syndrome. Clin Med 7: 53-56

3. Peri Y, Agmon-Levin N, Theodor E, Shoenfeld Y (2012)Sjögren's syndrome, the old and the new. Best Pract Res ClinRheumatol 26: 105-117.

4. Ebert EC (2012) Gastrointestinal and hepatic manifestations of Sjogren syndrome. J ClinGastroenterol46: 25-30

5. Vitali C Bombardieri S, Jonsson R, Moutsopoulos HM, Alexander EL, et al. (2002)Classification criteria for Sjogren's syndrome: a revised version of the European criteria proposed by the American-European Consensus Group. Ann Rheum Dis 61: 554-558.

6. Bernini P, Bertini I, Calabrò A, la Marca G, Lami G, et al. (2011)Are patients with potential celiac disease really potential? The answer of metabonomics. $J$ Proteome Res 10: 714-721

7. Bertini I, Calabrò A, De Carli V, Luchinat C, Nepi S, et al. (2009) The metabonomic signature of celiac disease. J Proteome Res 8: 170-177.

8. Macintyre DA, Jiménez B, Lewintre EJ, Martín CR, Schäfer H, et al. (2010) Serum metabolome analysis by ${ }^{1} \mathrm{H}-\mathrm{NMR}$ reveals differences between chronic lymphocytic leukaemia molecular subgroups. Leukemia 24:788-797.

9. Oakman C, Tenori L, Biganzoli L, Santarpia L, Cappadona S, et al. (2011a) Uncovering the metabolomic fingerprint of breast cancer. Int J Biochem Cell Biol 43:1010-1020.

10. Oakman C, Tenori L, Claudino WM, Cappadona S, Nepi S, et al. (2011b) Identification of a serum-detectable metabolomic fingerprint potentially correlated with the presence of micrometastatic disease in early breast cancer patients at varying risks of disease relapse by traditional prognostic methods. Ann Oncol 22: 1295-1301.

11. Tiziani S, Lopes V, Gunther UL (2009)Early stage diagnosis of oral cancer using ${ }^{1} \mathrm{H}$ NMR-based metabolomics. Neoplasia 11: 269-276.

12. Fleissig Y, Deutsch O, Reichenberg E, Redlich M, Zaks B, et al. (2009) Different proteomic protein patterns in saliva of Sjögren's syndrome patients. Oral Dis 15: 61-68

13. Giusti L, Baldini C, Bazzichi L, Ciregia F, Tonazzini I, et al.(2007)Proteome analysis of whole saliva: A new tool for rheumatic diseases - The example of Sjögren's syndrome. Proteomics 7:1634-1643.

14. Hu S, Wang J, Meijer J, leong S, Xie Y, et al. (2007)Salivary proteomic and genomic biomarkers for primary Sjögren's syndrome. Arthritis Rheum 56: 3588-3600.

15. Peluso G, De Santis M, Inzitari R, Fanali C, Cabras T, et al. (2007)Proteomic study of salivary peptides and proteins in patients with Sjögren's syndrome before and after pilocarpine treatment. Arthritis Rheum 56: 2216-2222.

16. Ryu OH, Atkinson JC, Hoehn GT, Illei GG, Hart TC (2006) Identification of parotid salivary biomarkers in Sjögren's syndrome by surface-enhanced lase desorption/ionization time-of-flight mass spectrometry and two-dimensional difference gel electrophoresis. Rheumatology 45: 1077-1086.

17. Harada H, Shimizu H, Maeiwa M (1987) ${ }^{1} H-N M R$ of human saliva. An application of NMR spectroscopy in forensic science. Forensic Scilnt 34:189-195

18. Grootveld M, Silwood CJL (2005) ${ }^{1} \mathrm{HNMR}$ analysis as a diagnostic probe for human saliva. BiochemBiophys Res Commun 329: 1-5.

19. Walsh MC, Brennan L, Malthouse JPG, Roche HM, Gibney MJ (2006)Effect of acute dietary standardization on the urinary, plasma, and salivary metabolomic profiles of healthy humans. Am J ClinNutr 84: 531-539.

20. Bertram HC, Eggers N, Eller N (2009) Potential of human saliva for nuclear magnetic resonance-based metabolomics and for health-related biomarker identification. Anal Chem 81: 9188-9193.

21. Takeda I, Stretch C, Barnaby P, Bhatnager K, Rankin K, et al. (2009) Understanding the human salivary metabolome. NMR Biomed 22: 577-584.

22. Silwood CJL, Lynch E, Claxson AWD, Grootveld M (2002) ${ }^{1} \mathrm{H}$ and ${ }^{13} \mathrm{C}$ NMR spectroscopic analysis of human saliva. J Dent Res 81: 422-427.

23. Aimetti M, Cacciatore S, Graziano A, Tenori L (2012) Metabonomicanalysis of saliva revealsgeneralizedchronic periodontitis signature. Metabolomics 8 : 465-474.

24. Niemelä RK, Takalo R, Pääkkö E, Suramo I, Päivänsalo M, et al. (2004) Ultrasonography of salivary glands in primary Sjögren's syndrome. A comparison with magnetic resonance imaging and magnetic resonance sialography of parotid glands. Rheumatology 43: 875-879. 
Citation: Mikkonen JJW, Herrala M, Soininen P, Lappalainen R, et al. (2013) Metabolic Profiling of Saliva in Patients with Primary Sjögren's syndrome. Metabolomics 3: 128. doi:10.4172/2153-0769.100012

Page 6 of 6

25. Seitsalo $\mathrm{H}$, Niemelä RK, Marinescu-Gava M, Vuotila T, Tjäderhane L, Salo $T$ (2007)Effectiveness of low-dose doxycycline (LDD) on clinical symptoms of Sjögren's Syndrome: a randomized, double-blind, placebo controlled crossover study. J Negat Results Biomed 6: 11-16.

26. NavazeshM (1993) Methods for collecting saliva. Ann NY AcadSci 694: 72-77

27. Soininen P, Haarala J, Vepsäläinen J, Niemitz M, Laatikainen R (2005) Strategies for organic impurity quantification by ${ }^{1} \mathrm{H}$ NMR spectroscopy: Constrained total-line-shape fitting. Anal ChimActa 542:178-185.

28. Liu J, Duan Y (2012) Saliva: A potential media fordiseasediagnostics and monitoring. Oral Oncol 48: 569-577.

29. Wong DT (2008)Salivary Diagnostics. Wiley-Blackwell: lowa

30. Helmerhorst EJ, Oppenheim FG (2007)Saliva: a dynamic proteome. J Den Res 86: 680-693.

31. Aframian DJ, Konttinen YT, Carrozzo M, Tzioufas AG (2013) Urban legends series: Sjögren's syndrome. Oral Dis 19:46-58.

32. de Almeida PDV, Grégio AMT, Machado MAN, de Lima AAS, Azevedo LR (2008) Saliva composition and functions: a comprehensive review. J Contemp Dent Pract 9:72-80.
33. Dawson LJ, Stanbury J, Venn N, HasdimirB, Rogers SN, et al. (2006) Antimuscarinic antibodies in primary Sjögren's syndrome reversibly inhibit the mechanism of fluid secretion by human submandibular salivary acinar cells. Arthritis Rheum 54: 1165-1173.

34. Hakala M, Niemelä RK (2000) Does autonomic nervous impairment have a role in pathophysiology of Sjögren's syndrome. Lancet 355: 1032-1033.

35. Perry M, Li Q, Kennedy RT (2009) Review of recent advances in analytical techniques for the determination of neurotransmitters. Anal ChimActa 653 $1-22$.

36. Deng YH,Wang H,Zhang HS(2008)Determinationofaminoacidneurotransmitters in human cerebrospinal fluid and saliva bycapillaryelectrophoresis with laserinducedfluorescencedetection. J SepSci31: 3088-3097.

37. Zinellu A, Sotgia S, Pisanu E, Scanu B, Sanna M, et al. (2010) Quantification of neurotransmitter amino acid by capillary electrophoresis laser-induced fluorescence detection in biological fluids. Anal Bioanal Chem 398: 1973-1978.

38. Greenmyre JT, Young AB (1989) Excitatory amino acids and Alzheimer's disease. Neurobiol Aging 10: 593-602.

39. Engelborghs S, Marescau B, De Deyn PP (2003) Amino acidsand biogenic amines in cerebrospinal fluid of patients with Parkinson's disease. Neurochem Res 28: 1145-1150. 